\title{
EMBEDDINGS INTO EFFICIENT GROUPS
}

\author{
by JENS HARLANDER
}

(Received 3rd August 1995)

\begin{abstract}
A finite presentation $F / N$ of a group $G$ is called efficient if $d_{F}(N)=d\left(H_{2}(G)\right)+d(F)-r\left(H_{1}(G)\right)$. A finitely presented group is called efficient if it admits an efficient presentation. We show that a finitely presented group embeds into an efficient group.
\end{abstract}

1991 Mathematics subject classification: $20 \mathrm{~F} 05$.

\section{Background}

If $A$ is a $G$-group, then $d_{G}(A)$ denotes the minimal number of $G$-group generators of $A$. For example the normal subgroup $N$ of a group $F$ is an $F$-group via conjugation and $d_{F}(N)$ is the minimal number of elements that generate $N$ as a normal subgroup. If $G$ acts trivially on $A$ we omit the subscript and simply write $d(A)$ for the minimal number of generators for the group $A$.

Given a finite presentation $\mathcal{P}=\langle X \mid R\rangle$ of a group $G$, let $F$ be the free group on $X$ and $N=N(R)$ be the normal closure of $R$ in $F$. Then $F / N=G$. We also refer to $F / N$ as a presentation for $G$. Now

$$
\text { (*) } \quad N /[F, N]=H_{2}(G) \oplus Z^{d(F)-r\left(H_{1}(G)\right)},
$$

where $r\left(H_{1}(G)\right)$ is the torsion free rank of the finitely generated abelian group $H_{1}(G)$. To see this, consider the exact 5 -term sequence

$$
H_{2}(F) \rightarrow H_{2}(G) \rightarrow N /[F, N] \rightarrow F /[F, F] \rightarrow G /[G, G] \rightarrow 0
$$

associated with the extension $N \rightarrow F \rightarrow G$ (see Brown [9, page 47]). Since $F$ is free, $H_{2}(F)=0$ and the result follows. In particular we have

$$
d(N /[F, N])=d\left(H_{2}(G)\right)+d(F)-r\left(H_{1}(G)\right) .
$$

For more details and additional references see Beyl, Tappe [5, page 18]. The presentation $\mathcal{P}=\langle X \mid R\rangle$ is called efficient if

$$
|R|=d_{F}(N)=d(N /[F, N]) .
$$


The group $G$ is called efficient if it admits an efficient presentation. Examples of efficient groups are finitely generated abelian groups (Epstein [13]), fundamental groups of closed 3-manifolds [13] and also finite groups with balanced presentations. Such finite groups have trivial Schur-multiplier. Whether finite groups with trivial Schur-multiplier are efficient (i.e., admit balanced presentations in this case) was answered negatively by Swan [26]. He gave examples of non-efficient metabelian groups with trivial $\mathrm{H}_{2}$. Finite metacyclic groups are efficient. This was shown by Wamsley [27] and Beyl [4]. Infinite metacyclic groups however need not be efficient, a result due to Baik and Pride [2] (see also Baik [1]). The first examples of torsion-free non-efficient groups were found by Lustig [21]. For more references on the subject of efficiency see Baik, Pride [3], Beyl, Rosenberger [6], Campbell, Robertson, Williams [10, 11], Johnson, Robertson [18], Kenne [20] and Robertson, Thomas, Wotherspoon [24].

Suppose $\langle X \mid R\rangle$ is a finite presentation for a group $H$. Assume that $u$ and $w$ are words in $X^{ \pm 1}$ and let $G$ be the quotient of $H$ presented by $\langle X \mid R, w\rangle$. Suppose the following conditions are satisfied:

1. $[u, w]$ represents the trivial element of $H$;

2. $u$ represents an element of infinite order of $G$;

3. The presentation $\langle X \mid R, w\rangle$ is efficient.

The group $G$ can be used to embed a given group into an efficient group by an iterated amalgamated product. Before we state our main result we introduce more notation. Let $S(K, G, l)$ be the fundamental group of a graph of groups supported by a graph with vertices $v, v_{1}, \ldots, v_{l}$ and oriented edges $e_{1}, \ldots, e_{l}$, where $e_{i}$ starts at $v$ and ends at $v_{i}$. The group at $v$ is $K$, all other vertex groups are $G$ (as above) and the edge groups are infinite cyclic. Edge maps are given by choosing elements of infinite order in $K$ and the other vertex groups.

Theorem. Suppose that $K$ is a finitely presented group that admits a generating set consisting of elements of infinite order. Suppose furthermore that, in case both $\mathrm{H}_{2}(\mathrm{~K})$ and $H_{2}(G)$ have torsion, the first torsion-numbers of these abelian groups are not relatively prime. Then there exists an integer l such that $S(K, G, l)$ is efficient.

Note that the condition on the torsion numbers ensures that $d\left(H_{2}(K) \oplus H_{2}(G)\right)=$ $d\left(H_{2}(K)\right)+d\left(H_{2}(G)\right)$.

There is considerable flexibility in choosing $G$. For example we can take $H=\left\langle a, b \mid a^{n}=b^{n}\right\rangle, u=a b$ and $w=a^{n}$. In that case we get $G=\left\langle a, b \mid a^{n}=b^{n}, a^{n}\right\rangle=Z_{n} * Z_{n}$, the free product of two cyclic groups of order $n$. Or we could take $H=$ $\langle a, b, c \mid[a, b],[a, c]\rangle, u=a$ and $w=[b, c]$. Here we obtain $G=\langle a, b, c \mid[a, b],[a, c],[b, c]\rangle=$ $Z \oplus Z \oplus Z$. Note that in both cases $H_{2}(G)$ is torsion-free. Before we prove the Theorem we point out some consequences.

Corollary 1. Let $K$ be a finitely presented group and let $d(K)=k$. Let $F_{k}$ be the free 
group of rank $k$. Then there exists an integer l such that $S\left(K * F_{k}, Z_{n} * Z_{n}, l\right)$ is efficient. In particular a finitely presented group can be embedded into a finitely presented efficient group.

Proof. Let $y_{1}, \ldots, y_{k}$ be a set of generators for $K$ and let $a_{1}, \ldots, a_{k}$ be a basis for $F_{k}$. Then $y_{1} a_{1}, \ldots, y_{k} a_{k}, a_{1}, \ldots, a_{k}$ is a generating set of $K * F_{k}$ consisting of elements of infinite order. Now apply the Theorem.

We remark that the author showed in [15] that a finite group can be embedded into a finite efficient group. In fact, if $K$ is finite, then $K \times \prod_{i=1}^{l} Z_{p}$ is efficient for $l$ big enough and $p$ a prime.

Corollary 2. Let $K$ be a finitely presented group of finite cohomological dimension $k$. If $k \neq 2$, then $K$ can be embedded into an efficient group of cohomological dimension $k$. If $k=2$, then $K$ can be embedded into an efficient group of virtual cohomological dimension 2.

Proof. If $k=1$ then, by Stallings' Theorem [25], $K$ is free and thus itself efficient. So suppose $k \geq 2$. Since $K$ is torsion-free, it admits a generating set consisting of elements of infinite order. We can apply the Theorem to see that $\tilde{G}=S(K, G, l)$ is efficient for big enough $l$ and an appropriately chosen group $G$. If $k=2$ take $G=Z_{n} * Z_{n}$. The virtual cohomological dimension of both $Z$ and $Z_{n} * Z_{n}$ is one and hence $v c d(\tilde{G})=v c d(K)=2$ (see Bieri [7, page 83]). If $k \geq 3$ take $G=Z \oplus Z \oplus Z$. Since $c d(G)=3$ it follows that $c d(\tilde{G})=c d(K)=k$.

Whether a group of cohomological dimension 2 can be embedded into an efficient group of cohomological dimension 2 is related to the question whether a group of cohomological dimension 2 has geometric dimension 2. A discussion of these matters can be found in Section 4 of this paper.

\section{The main lemma}

The proof of the main theorem in this article is based on an idea of Wolfgang Metzler. He realized (see [23]) that wedging on standard 2-complexes of $Z_{2} \times Z_{4}$ to a given 2-complex allows one to bypass the commutator question, a serious obstruction encountered when attempting to generalize results from higher dimensions into dimension 2. Hog-Angeloni and Metzler have successfully applied this trick to various situations (see Metzler [23] and also Metzler, Hog-Angeloni [16]). We will present a generalized $Z_{2} \times Z_{4}$ trick, which is tailored to our situation. Suppose $\mathcal{P}_{H}=\langle X \mid R\rangle$ is a finite presentation for the group $H$ and $u$ and $w$ are words in $X^{ \pm 1}$ so that the commutator $[u, w]$ represents the trivial element of $H$. Let $G$ be the quotient of $H$ represented by $\mathcal{P}_{G}=\langle X \mid R, w\rangle$. Let $n$ be the order of the element of $G$ represented by $u$ (the order can be infinite). Next assume that $K$ is another group admitting a finite 
presentation $\mathcal{P}_{K}=\langle Y \mid S,[f, t]\rangle$, where $t$ is a consequence of $S,[f, t]$ in $\mathcal{P}_{K}$, that is $t \in N(S,[f, t])$, and $f$ represents an element of order $n$ in $K$. We can form the free product with amalgamation $\tilde{G}=K *_{z_{n}} G$ with presentation $\mathcal{P}=\langle X, Y| R, w, S$, $[f, t], u=f\rangle$. The key observation here is that the normal closure of the relations in $\mathcal{P}$ is generated by $|R|+1+|S|+1$ elements, which is one less than expected. Indeed $\{R, S, w=t, u=f\}$ is a generating set for that normal closure. Just observe that

$$
[f, t]=[u, w]=1
$$

modulo the relations $f=u, t=w$ and $R$. Since we assumed that $t=1$ modulo $S$ and $[f, t]$, this shows that $w=1$ modulo $R, S, f=u$ and $t=w$. If we iterate the above process we obtain the following.

Lemma. Suppose $K$ is a group admitting a presentation $\mathcal{P}_{K}=\left\langle Y \mid S,\left[f_{i}, t_{i}\right]\right\rangle, 1 \leq i \leq l$, where each $t_{i}$ is contained in $N\left(S,\left[f_{1}, t_{1}\right], \ldots,\left[f_{1}, t_{1}\right]\right)$, and each $f_{i}$ represents an element of infinite order. Let

$$
\mathcal{P}=\left\langle X_{i}, Y \mid S,\left[f_{i}, t_{i}\right], R_{i}, w_{i}, u_{i}=f_{i}\right\rangle,
$$

$1 \leq i \leq l,\left\langle X_{i} \mid R_{i}, w_{i}\right\rangle$ presenting $G, u_{i}$ representing an element of infinite order in $G$, be the standard presentation for the amalgamated product $S(K, G, l)$. Then the normal closure of the relations in $\mathcal{P}$ is generated by $|S|+(|R|+2)$ l elements.

A free product version of the above Proposition with $Z_{2} \times Z_{4}$ factors is implicit in [23], dealing with commutators of relators, that is with elements of $[N, N]$ rather than $[F, N]$.

\section{Proof of the theorem}

Let $F / N$ be a finite presentation for the group $K$, where $F$ is a free group with basis $Y$ and each element $y$ of $Y$ represents an element of infinite order in $K$. Let $m=d(N /[F, N])$. We can find elements $s_{1}, \ldots, s_{m}$ of $N$ so that $s_{1}[F, N], \ldots, s_{m}[F, N]$ generates $N /[F, N]$. Since $N$ is the normal closure of finitely many elements, we can find elements $f_{i} \in F, t_{i} \in N, 1 \leq i \leq l$, so that $N=N\left(s_{1}, \ldots, s_{m},\left[f_{i}, t_{i}\right]\right)$. Thus

$$
\mathcal{P}_{K}=\left\langle Y \mid s_{1}, \ldots, s_{m},\left[f_{i}, t_{i}\right]\right\rangle,
$$

$1 \leq i \leq l$, presents $K$. Note that because $\left\{\left[y^{ \pm 1}, r\right] \mid y \in Y, r \in N\right\}$ generates $[F, N]$ we may assume that each $f_{i}$ is equal to some $y^{ \pm 1}$, in particular that each $f_{i}$ has infinite order in $K$. Let $\mathcal{P}_{G}=\langle X \mid R, w\rangle$ be an efficient presentation of a group $G$ as in the previous section. Then we have a word $u$ in $X^{ \pm 1}$ representing an element of infinite order in $G$ and $[u, w]=1$ modulo $R$. Let 


$$
\mathcal{P}=\left(X_{i}, Y\left|S,\left[f_{i}, t_{i}\right], R_{i}, w_{i}, f_{i}=u_{i}\right\rangle,\right.
$$

$1 \leq i \leq l$, be the standard presentation for the amalgamated product $S(K, G, l)$ as in the Lemma. Let $\tilde{F}$ be the free group on the generators in $\mathcal{P}$ and let $\tilde{N}$ be the normal closure of the relations in $\mathcal{P}$. Furthermore let $F_{i}$ be the free group on $X_{i}$ and let $N_{i}$ be the normal closure of $R_{i}$ and $w_{i}$ in $F\left(X_{i}\right)$. So $F_{i} / N_{i}$ presents the vertex group $G$ at $v_{i}$ in the above amalgamated product. We know from the Lemma that $d_{F}(\tilde{N}) \leq$ $m+(|R|+2) l$. We claim that $d(\tilde{N} /[\tilde{F}, \tilde{N}])=m+(|R|+2) l$ and thus that $\tilde{F} / \tilde{N}$ is efficient. Before we show this, let us make some general remarks. Suppose $F_{i} / N_{i}$ is a finite presentation for $G_{i}, i=1,2$, and that $C$ is a finitely generated subgroup of both $G_{1}$ and $G_{2}$. Let $F / N$ be a presentation for the amalgamated product $G=G_{1} *_{C} G_{2}$, obtained from the presentations $F_{i} / N_{i}$ and a fixed finite generating set for $C$. Then we have an exact sequence (see Hannerbauer [14])

$$
0 \rightarrow\left(Z G \otimes_{G_{1}} N_{1} /\left[N_{1}, N_{1}\right]\right) \oplus\left(Z G \otimes_{G_{2}} N_{2} /\left[N_{2}, N_{2}\right]\right) \rightarrow N /[N, N] \rightarrow Z G \otimes_{C} I C \rightarrow 0 .
$$

If we apply $Z \otimes_{G}-$ we obtain the exact sequence

$$
H_{2}(C) \rightarrow N_{1} /\left[F_{1}, N_{1}\right] \oplus N_{2} /\left[F_{2}, N_{2}\right] \rightarrow N /[F, N] \rightarrow H_{1}(C) \rightarrow 0 .
$$

In case $C$ is infinite cyclic, $H_{2}(C)=0$ and $H_{1}(C)=Z$ and we obtain

$$
N /[F, N]=N_{1} /\left[F_{1}, N_{1}\right] \oplus N_{2} /\left[F_{2}, N_{2}\right] \oplus Z .
$$

If we apply this result to our presentation $\tilde{F} / \tilde{N}$ of $S(K, G, l)$ we get

$$
\tilde{N} /[\tilde{F}, \tilde{N}]=N /[F, N] \oplus \bigoplus_{i=1}^{\prime} N_{i} /\left[F_{i}, N_{i}\right] \oplus Z^{\prime} .
$$

This follows from the above discussion and induction on $l$ since

$$
S(K, G, l)=S(K, G, l-1) *_{C} G,
$$

with $C$ infinite cyclic. Since $F_{i} / N_{i}=\left\langle X_{i} \mid R_{i}, w_{i}\right\rangle$ is an efficient presentation for $G$, we have $d\left(N_{i} /\left[F_{i}, N_{i}\right]\right)=|R|+1$. Since the first torsion-numbers of $H_{2}(G)$ and $H_{2}(K)$ are not relatively prime (in case both $H_{2}(K)$ and $H_{2}(G)$ contain torsion), we have $d\left(H_{2}(K) \oplus H_{2}(G)\right)=d\left(H_{2}(K)\right)+d\left(H_{2}(G)\right)$. Since $N /[F, N]$ is the direct sum of $H_{2}(K)$ and a free abelian group and each $N_{i} /\left[F_{i}, N_{i}\right]$ is the direct sum of $H_{2}(G)$ and a free abelian group (see equation $(*)$ on the first page), we have

$$
d(\tilde{N} /[\tilde{F}, \tilde{N}])=d(N /[F, N])+\sum_{i=1}^{l} d\left(N_{i} /\left[F_{i}, N_{i}\right]\right)+l .
$$

Hence $d(\tilde{N} /[\tilde{F}, \tilde{N}])=m+(|R|+2) l$ as claimed. 
JENS HARLANDER

\section{Groups of dimension 2}

A group $G$ has cohomological dimension 2 if the trivial $G$-module $Z$ admits a projective resolution of length 2 . The geometric dimension of $G$ is 2 if there exists a 2-dimensional $K(G, 1)$-complex. A group $G$ is of type $F L$ if $Z$ admits a resolution of finite length consisting of finitely generated free $Z G$-modules. A presentation $\mathcal{P}$ is aspherical if the associated 2-complex $K(\mathcal{P})$ modelled on $\mathcal{P}$ is aspherical (that is, it has trivial second homotopy group). Note that in that case $K(\mathcal{P})$ is a $K(G, 1)$-complex and thus $G$ and all it's subgroups have geometric dimension 2 . These definitions can be found in [9].

Efficient groups of cohomological dimension 2 are of interest in connection with the longstanding open question whether cohomological dimension 2 implies geometric dimension 2. The next proposition shows that subgroups of an efficient group of cohomological dimension 2 that is $F L$ have geometric dimension 2 . This result is due to Gutierrez and Ratcliffe [17] (see also Bogley [8, page 329]). In [17] it is stated for subcomplexes of aspherical complexes. Such complexes give rise to presentations which are not only efficient but satisfy the Cockcroft property (see [12, page 149]). For the convenience of the reader we have also included a proof.

Proposition. Let $\mathcal{P}$ be a finite presentation of a group $G$ of cohomological dimension 2 that is of type $F L$. Then $\mathcal{P}$ is efficient if and only if $\mathcal{P}$ is aspherical.

Proof. Let $\mathcal{P}=\left\langle x_{1}, \ldots, x_{n} \mid r_{1}, \ldots, r_{m}\right\rangle$ be an efficient presentation for $G$. Let $F$ be the free group on the generators in $\mathcal{P}$ and let $N$ be the normal closure of the relations of $\mathcal{P}$ in $F$. Cohomological dimension 2 together with $F L$ implies that the relation module $N /[N, N]$ of this presentation is finitely generated stably free. This is a consequence of Schanuel's Lemma (see [9, page 192]). So suppose $N /[N, N] \oplus Z G^{k}=Z G^{\prime}$. Replacing $\mathcal{P}$ with $\left\langle x_{1}, \ldots, x_{n}, y_{1}, \ldots, y_{k} \mid r_{1}, \ldots, r_{m}, y_{1}, \ldots, y_{k}\right\rangle$, we obtain an efficient presentation with free relation module of rank $l$. In particular $l=m+k$. Since the 2-complex associated with the modified presentation is simply homotopic to the two complex associated with $\mathcal{P}$, asphericity of the new presentation implies asphericity of $\mathcal{P}$. This discussion shows that we may assume that the relation module of $\mathcal{P}$ is free of rank $m$. Let us look at the partial resolution (see Lyndon, Schupp [22, page 100])

$$
\pi_{2}(K(\mathcal{P})) \rightarrow Z G^{m} \stackrel{\partial_{2}}{\rightarrow} Z G^{n} \stackrel{\partial_{1}}{\longrightarrow} Z G \rightarrow Z \rightarrow 0
$$

associated with $\mathcal{P}$ (it arises from the cellular chain complex of the universal covering of $K(\mathcal{P})$ ). The image of the boundary map $\partial_{2}$ is the relation module which is free of rank $m$. Thus it follows from Kaplansky's Theorem (see [19], and also [8, page 328]) that $\partial_{2}$ is an isomorphism and that $\pi_{2}(K(\mathcal{P}))$ is trivial. Thus $\mathcal{P}$ is an aspherical presentation for $G$. This proves one direction. That asphericity of a presentation implies efficiency is immediate from the partial resolution associated with $\mathcal{P}$. 
The property $F L$ was needed to ensure that every finite presentation of $G$ has stably free relation module. It should be noted that there are no examples known of finitely presented groups of cohomological dimension 2 that are not $F L$. We know from Corollary 2 of Section 1 that a finitely presented group $K$ of cohomological dimension 2 can be embedded into an efficient group $S\left(K, G=Z_{n} * Z_{n}, l\right)$, which is of virtual cohomological dimension 2. If we could replace $Z_{n} * Z_{n}$ by a group $G$ of cohomological dimension 2 for which our method works, we could eliminate "virtual". If in addition $S(K, G, l)$ is $F L$, then $K$ is actually of geometric dimension 2 by the above Proposition. But we believe that such a group $G$ is difficult to find. For our techniques to work we would have to find an efficient presentation $\mathcal{P}=\langle X \mid R, w\rangle$ of a group $G$ of cohomological dimension 2 and a word $u$ representing an element of infinite order such that $[u, w]=1$ modulo $R$. Thus we would have an identity of relations $u w u^{-1} w^{-1} \prod_{i=1}^{k} f_{i} r_{j_{i}}^{\epsilon_{i}} f_{i}^{-1}=1, f_{i}$ words in $X^{ \pm 1}, \epsilon_{i} \in\{ \pm 1\}, r_{j_{i}} \in R$, which yields a non-trivial spherical element over $\mathcal{P}$ since $u$ is not trivial. So $\mathcal{P}$ is an efficient non-aspherical presentation of a group $G$ of cohomological dimension 2. In view of the above Proposition, $G$ could not be $F L$ !

Of course the group $S\left(K, Z_{n} * Z_{n}, l\right)$ contains a torsion-free subgroup of finite index of cohomological dimension 2 . We conclude by remarking that a subgroup of finite index of an efficient group need not be efficient. It was shown in [15] that a finite group can be embedded into a finite efficient group. Since there are non-efficient finite groups (Swan's examples for instance), finite index does not preserve efficiency.

\section{REFERENCES}

1. Y. G. BAIK, Generators of the second homotopy module of group presentations with applications (Ph.D. thesis, Glasgow University, 1992).

2. Y. G. BAIK and S. J. PRIDE, Generators of the second homotopy module of presentations arising from group constructions, preprint, Glasgow University, 1993.

3. Y. G. BaIK and S. J. PRIDE, On the efficiency of Coxeter-groups, preprint, University of Glasgow, 1995.

4. F. R. BeYL, The Schur-multiplicator of metacyclic groups, Proc. Amer. Math. Soc. 40 (1973), 413-418.

5. F. R. BeYL and J. TAPPE, Group Extensions, Representations, and the Schur Multiplicator (Lecture Notes in Mathematics 958, Springer-Verlag, 1982).

6. F. R. BeyL and G. Rosenberger, Efficient presentations of $G L(2, Z)$ and $P G L(2, Z)$, in Groups-St Andrews 1985 (E. F. Robertson and C. M. Campbell eds, LMS Lecture Notes 121, Cambridge University Press 1986), 135-137.

7. R. BIERI, Homological Dimension of Discrete Groups (Queen Mary College Mathematics Notes, London, 1976).

8. W. A. Bogley, J. H. C. Whiteheads's asphericity question, in Two-dimensional Homotopy and Combinatorial Group Theory (edited by C. Hog-Angeloni, W. Metzler and A. J. Sieradski, LMS Lecture Notes 197, Cambridge University Press 1993).

9. K. Brown, Cohomology of Groups (Graduate Texts in Mathematics 87, Springer, New York, 1982). 
10. C. M. Campbell, E. F. Robertson and P. D. Williams, Efficient presentations for finite simple groups and related groups, in Groups-Korea 1988 (A. C. Kim and B. H. Neumann eds, Lecture Notes in Mathematics 1398, Springer-Verlag, 1989), 65-72.

11. C. M. Campbell, E. F. Robertson and P. D. Williams, On the efficiency of some direct powers of groups, in Groups-Canberra 1989 (L. G. Kovacs ed, Lecture Notes in Mathematics 1456, Springer-Verlag, 1990), 65-72.

12. M. N. DYer, Crossed modules and $\pi_{2}$-homotopy modules, in Two-dimensional Homotopy and Combinatorial Group Theory (edited by C. Hog-Angeloni, W. Metzler and A. J. Sieradski, LMS Lecture Notes 197, Cambridge University Press 1993).

13. D. B. A. EpSTEIN, Finite presentations of groups and 3-manifolds, Quart. J. Math. Oxford (2) 12 (1961), 205-212.

14. T. Hannerbauer, Relation modules of amalgamated free products and $H N N$-extentions, Glasgow Math. J. 31 (1989), 263-270.

15. J. Harlander, Closing the relation gap by direct product stabilization, J. Algebra, to appear.

16. C. Hog-Angeloni and W. Metzler, Stabilization by free products giving rise to Andrews-Curtis equivalence, Note Mat. 10, Supp. n. 2 (1990), 305-314.

17. M. A. Gutierrez and J. G. RatCliffe, On the second homotopy group, Quart. J. Math. Oxford (2) 32 (1981), 45-55.

18. D. L. Johnson and E. F. Robertson, Finite groups of deficiency zero, in: Homological Group Theory (C. T. C. Wall ed, LMS Lecture Notes Series 36, Cambridge University Press 1979).

19. I. KA PLANSKY, Fields and Rings (University of Chicago Press 1972).

20. P. E. KenNe, Some new efficient soluble groups, Comm. Algebra 18 (1990), 2747-2753.

21. M. Lustig, Fox-ideals, $N$-torsion and applications to groups and 3 -manifolds, in $T w o$ Dimensional Homotopy and Combinatorial Group Theory (edited by C. Hog-Angeloni, W. Metzler and A. Sieradski, London Math. Soc. Lecture Notes 197, Cambridge University Press, 1993).

22. R. C. LYNDON and P. E. SCHUPP, Combinatorial Group Theory (Springer-Verlag, BerlinHeidelberg-New York, 1977).

23. W. MetzleR, Die Unterscheidung von Homotopie-Typ und einfachem Homotopie-Typ bei 2-dimensionalen Komplexen, J. Reine Angew. Math. 403 (1990), 201-219.

24. E. F. Robertson, R. M. Thomas and C. I. Wotherspoon, A class of inefficient groups with symmetric presentations, preprint, University of St Andrews, 1994.

25. J. R. Stallings, On torsion-free groups with infinitely many ends, Ann. of Math. 88 (1968), 312-334.

26. R. G. Swan, Minimal resolutions for finite groups, Topology 4 (1985), 193-208.

27. J. W. WAmsley, The deficiency of metacyclic groups, Proc. Amer. Math. Soc. 24 (1970), 724-726.

FB Mathematik

UNIVERSITÄT FRANKFURT

ROBERT-MAYER-STR. 8

60054 FrANKFURT/MAIN

GeRManY 